\title{
Effect of Low-Level Electrical Stimulation of the Arotic Root Ventricular Ganglionated Plexi on Electrical and Structural Remodling in Dogs with Heart
}

\section{Failure}

\author{
Hong-Tao Wang, Fei-Fei Su, Di Zeng, Boyuan-Fan, Jun \\ Li and Qiang-sun Zheng
}

Division of Cardiology, Tangdu Hospital, Fourth Military Medical University, Xi'an 710038, China
${ }^{*}$ Corresponding author

Qiang-Sun Zheng, Division of Cardiology, Tangdu Hospital, Fourth Military Medical University, Xi'an 710038, China, Tel: 86029- 84777422; Fax: 86-029- 84777422; E-mail: qiangsun_tdxn@ hotmail.com.

Submitted: 10 Nov 2016; Accepted: 21 Nov 2016; Published: 24 Nov 2016

\begin{abstract}
Low-level electrical stimulation (LL-ES) of aortic root ventricular ganglionated plexi (GP) was proved to be antiarrhythmic in the initiation of AF mediated by autonomic nervous system. However,it is still uncertain whether $L L-E S$ of the ventricular GP can reverse the structural remodeling of myocardial fibrosis and atrial enlargement following heart failure by attenuating the sympathetic tone. Therefore, this review will give an general argument on this topic.
\end{abstract}

The rise of vagal tone facilitated the activity of pulmonary veins and played an important role in the initiation of AF [1]. Autonomic nerve stimulation shortens AERP, decreases the wavelength of atrial reentrant circuits and increases dAERP, thus, promoting the stability of AF [2]. Several studies have demonstrated that additional ablation of epicardiol ganglionated plexi (GP) improved success rate in catheter ablation of AF after circumferential isolation of pulmonary veins $[3,4]$. But in long term follow-up, the effect of GP ablation is limited due to high reoccurance rate of AF $[5,6]$.

As for more than a century, basic scientists had used strong vagal stimulation, which slowed the heart rate or caused sinus arrest, as a method for inducing AF. Recently, Li et al. gave a novel idea that low-level electrical stimulation (LL-ES) of vagal nerve VNS could suppress AF by inhibiting the intrinsic cardiac autonomic nervous system (ANS) and prevent episodic AF caused by rapid pulmonary vein and non-pulmonary vein firing [7]. Following Li's study, increasing evidences suggested that LL-ES of vagal nerve, with voltage levels $10-50 \%$ below threshold showed an antiarrhythmic effect [8-10]. Recently, Stavros Stavrakis [11] applied LL-ES skill to treat patients with paroxysmal atrial fibirlaltion, and they demonstrated that transcutaneous LL-ES suppresses AF and decreases inflammatory cytokines in patients with paroxysmal AF. These results indicated LL-ES of automomic nerve could bring both anti- arrhythmia and anti-inflammation effect.

Since intrinsic ANS are mainly connected by some GPs that located in the epicardiol fat pads. Previous study proved that epicardial GP could be anatomically divided into the atrial and ventricular GP, and the ventricular GP mainly innervated the ventricle and coronary artery [12]. Moreover, He et al. found that the activity of atrial GP influenced the electrophysiology of the ventricle and ventricular arrhythmogenic properties as well [13,14]. These results indicated that the atrial and ventricular GP may cooperate with each other and act as a single functional unit. Therefore, we proposed that modulation of the ventricular GP would also affect the electrophysiology of the atrium. Our recent study demonstrated that stimulation of aortic root ventricular GP provoked robust $\mathrm{AF}$ in the absence of extrinsic cardiac nerve activity using an isolated perfused heart model [15]. These findings suggested that ventricular GP innervated PVs and contribute to the initiation of AF with the exception of atrial GP. Moreover, our latest studies showed that LL-ES of the aortic root ventricular GP attenuated the tension of autonomic nerves and reduce the occurrence of AF mediated by autonomic nervous system, which demonstrated the antiarrhythmic effect of LL-ES of the aortic root ventricular GP [16].

Because arrhythmia, especially AF is one of the complications accompanied with HF. AF induces atrial enlargement and electrical remodeling, which aggravates HF. It has been revealed that myocardial fibrosis and electrical remodeling resulted from high sympathetic tone offered an essential substrate for the development and maintenance of AF [17,18]. Previous studies also proved that modulation of vagal tone can reverse electrical or functional remodeling of $\mathrm{HF}$. $\mathrm{Li}$ stated that vagal nerve stimulation can modulate the inflammatory response and affect specific inflammatory mediators including nitric oxide that may be contributory to continued or progressive heart failure [19]. Therefore, vagal nerve stimulation may present beneficial effects that are independent from heart rate or AV conduction in 
heart failure. Kobayashi et al. found that endovascular cardiac GP stimulation induced significant and selective increases in left ventricule contractility without increasing heart rate [20]. Efforts to optimize electrode placement and fixation will improve the reproducibility of endovascular cardiac GP stimulation. These studies demonstrated that the decrease tone of ANS could also benefit to the treat of HF. It is therefore plausible to hypothesize that LL-ES of the aortic root ventricular GP would be antiarrhythmic and anti-inflammation, which might reverse the structural remodeling of myocardial fibrosis and atrial enlargement following HF by attenuating the sympathetic tone.

Therefore, our further study will use programmed electrical stimulation, burst electrical stimulation, immunohistochemistry, PCR and Western Blot, Ellesa, and patch clamp skills to determine the following data's. First, bioactive factors for HF, such as angiotensin II, TGF- $\beta$, mitogen-activated protein kinase (MAPK), matrix metalloproteinase (MMP) and tissue inhibitors of matrix metalloproteinase (TIMP) will be assessed to explore whether long-term LL-ES of the aortic root ventricular GP is capable of reversing the structural remodeling of the heart and help to treat HF. Second, ventricular effective refractory periods (VERPs), the dispersion of VERP and the inducing rate of arrhythmia by programmed electrical stimulation will also be measured to determine whether long-term LL-ES of the aortic root ventricular GP would improve the electrophysiological properties of the ventricle and get an antiarrhythmic effect.

\section{References}

1. Chen PS, Tan AY (2007) Autonomic nerve activity and atrial fibrillation. Heart Rhythm 4: S61-64.

2. Li Y, Li W, Yang B, Han W, Dong D, et al. (2007) Effects of Cilazapril on atrial electrical, structural and functional remodeling in atrial fibrillation dogs. J Electrocardiol 40: 100.

3. Platt M, Man dapati R, Scherlag BJ (2004) Limiting the number and extent of radiofrequency applications to terminate atrial fibrillation and subsequently prevent its inducibility (abstract). Heart Rhythm 1: S11.

4. Pappone C, Santinelli V, Manguso F, Vicedomini G, Gugliotta F, et al. (2004) Pulmonary vein denervation enhances longterm benefit after circumferential ablation for paroxysmal atrial fibrillation. Circulation 109: 327-334.

5. Mikhaylov E, Kanidieva A, Sviridova N, Abramov M, Gureev $\mathrm{S}$, et al. (2011) Outcome of anatomic ganglionated plexi ablation to treat paroxysmal atrial fibrillation: a 3-year followup study. Europace 13: 362-370.

6. Sakamoto S, Schuessler RB, Lee AM, Aziz A, Lall SC, et al. (2010) Vagal denervation and reinnervation after ablation of ganglionated plexi. J Thorac Cardiovasc Surg 139: 444-452.

7. Shuyan Li, Scherlag BJ, Yu Lilei, Sheng Xia, Zhang Ying, et al. (2009) Low-level vagosympathetic stimulation a paradox and potential new modality for the treatmentof facal atrial fibrillation. Circ Arrhythm Electrophysol 2: 645-651.

8. Chen M, Zhou X, Liu Q, Sheng X, Yu L, et al. (2015) Leftsided Noninvasive Vagus Nerve Stimulation Suppresses Atrial Fibrillation by Up-regulating Atrial Gap Junctions in Canines.
J Cardiovasc Pharmacol 66: 593-599.

9. Stavrakis S, Nakagawa H, Po SS, Scherlag BJ, Lazzara $\mathrm{R}$, et al. (2015) The role of the autonomic ganglia in atrial fibrillation. JACC Clin Electrophysiol 1: 1-13.

10. Liao K, Yu L, Zhou X, Saren G, Wang S, et al. (2015) Lowlevel baroreceptor stimulation suppresses atrial fibrillation by inhibiting ganglionated plexus activity. Can J Cardiol 31: 767774.

11. Stavrakis Humphrey MB, Scherlag BJ, Hu Y, Jackman WM, Nakagawa H, et al. (2015) Low-Level Transcutaneous Electrical Vagus Nerve Stimulation Suppresses Atrial Fibrillation. J Am Coll Cardiol 9: 867-875.

12. Armour JA, Murphy DA, Yuan BX, Macdonald S, Hopkins DA (1997) Gross and microscopic anatomy of the human intrinsic cardiac nervous system. Anat Rec 247: 289-298.

13. He B, Lu Z, He W, Huang B, Yu L, et al. (2013) The effects of atrial ganglionated plexi stimulation on ventricular electrophysiology in a normal canine heart. J Interv Card Electrophysiol 37: 1-8.

14. He B, Lu Z, He W (2013) Effects of ganglionated plexi ablation on ventricular electrophysiological properties in normal hearts and after acutemyocardial ischemia. Int J Cardiol 168: 86-93.

15. Hong-Tao Wang, Zhi-Li Li, Bo-Yuan Fan, Fei-Fei Su, JinBo Zhao, et al. (2014) The independent role of the aortic root ganglionated plexi in the initiation of atrial fibrillation: an experimental study. J Thorac Cardiovasc Surg 148: 73-76.

16. Hong-Tao Wang, Ming Xu, Boyuan Fan, Xiong-Tao Liu, FeiFei Su, et al. (2015) Low-level electrical stimulation of aortic root ventricular ganglionated plexi attenuates autonomic nervous system-mediated atrial fibrillation. JACC Clin Electrophysiol 1: 390-397.

17. Tang RB, Yan XL, Dong JZ, Kalifa J, Long DY, et al. (2014) Predictors of recurrence after a repeat ablation procedure for paroxysmal atrial fibrillation: role of left atrial enlargement. Europace 16: 1569-1574.

18. De Jong AM, Maass AH, Oberdorf-Maass SU (2011) Mechanisms of atrial structural changes caused by stretch occurring before and during early atrial fibrillation. Cardiovasc Res 89: 754-765.

19. Li W, Olshansky B (2011) Inflammatory cytokines and nitric oxide in heart failure and potential modulation by vagus nerve stimulation. Heart Fail Rev 16: 137-145.

20. Kobayashi M, Sakurai S, Takaseya T, Shiose A, Kim HI, et al. (2012) Effects of percutaneous stimulation of both sympathetic and parasympathetic cardiac autonomic nerves on cardiac function in dogs. Innovations (Phila) 7: 282-289.

Copyright: (02016 Qiang-sun Z, et al. This is an open-access article distributed under the terms of the Creative Commons Attribution License, which permits unrestricted use, distribution, and reproduction in any medium, provided the original author and source are credited. 